\title{
Coupling of quantum degrees of freedom in strongly interacting disordered electron systems
}

\author{
V. Janiš and D. Vollhardt \\ Institut für Theoretische Physik C, Technische Hochschule Aachen, D-5100 Aachen, Federal Republic of Germany
}

(Received 8 September 1992)

\begin{abstract}
We derive the exact expression for the averaged free energy of a general class of Hubbard-type models with local, quenched disorder in the limit of high dimensions. A clear mean-field interpretation of its structure is given. Analytic results are obtained for a simplified Hubbard model with disorder. The two-particle correlation functions are shown to display a novel coupling of quantum degrees of freedom whenever genuine electron-electron correlation effects are present.
\end{abstract}

Many essential properties of doped semiconductors near the metal-insulator transition, of high- $T_{c}$ superconducting materials close to $T_{c}$, and of other systems, are determined by the simultaneous presence of strong electron-electron interactions and disorder. However, a detailed microscopic explanation of the interplay between these correlation effects and its influence on the thermodynamic and transport properties of itinerant electron systems still does not exist. During the last ten years, various aspects of this problem have been investigated by different methods. ${ }^{1}$ In particular, the application of renormalization-group techniques to a field-theoretical model (nonlinear $\sigma$ model $^{2}$ ), has led to promising progress. ${ }^{3,4}$ The appearance of local magnetic moments that lead to serious complications in this approach has been examined within an effective-field theory. ${ }^{5}$ Moreover, the competition between strong interactions and disorder has been studied by means of the Hubbard model with local disorder: while $\mathrm{Ma}^{6}$ applied a real-space renormalization group, Zimanyi and Abrahams ${ }^{7}$ recently used a slaveboson formulation of the $t-J$ model to calculate the phase diagram.

The above results provide insight into important partial aspects of the problem of disordered, interacting electrons. To obtain a global picture of the properties of such systems, it would be desirable to know a solution of a simple, microscopic model which is valid for all input parameters (interaction, disorder, temperature, band filling). Since exact solutions cannot be obtained in $d=2,3$ one would, at least, like to construct a thermodynamically consistent mean-field theory. Such a (nonperturbative) approximation is provided by the exact solution of a model in $d=\infty$. In this limit, ${ }^{8}$ any nonlocal interaction reduces exactly to the Hartree contribution and only the on-site interaction remains dynamical; ${ }^{9}$ indeed, the theory then reduces to an effective single-site problem. ${ }^{10-12}$ For noninteracting, disordered electrons, the results of the coherent potential approximation (CPA) become exact. ${ }^{13}$

In this paper we first construct the exact averaged free energy of a general class of Hubbard-type models with local disorder in $d=\infty$. We then deduce various correlation functions that allow us to gain new insight into the properties of interacting, disordered electron systems. We start from the Hamiltonian

$$
\hat{H}=-\sum_{\langle i j\rangle} \sum_{\sigma} t_{\sigma} \hat{c}_{i \sigma}^{\dagger} \hat{c}_{j \sigma}+\frac{1}{2} \sum_{i, j} \sum_{\sigma, \sigma^{\prime}} V_{i j}^{\sigma \sigma^{\prime}} \hat{n}_{i \sigma} \hat{n}_{j \sigma^{\prime}}+\sum_{i, \sigma} \hat{v}_{i \sigma} \hat{n}_{i \sigma}
$$

where the interaction is expressed in terms of a general off-diagonal term $V_{i j}^{\sigma \sigma^{\prime}}$, and a local potential-energy operator $\hat{v}_{i \sigma}=\frac{1}{2} U \hat{n}_{i,-\sigma}+\epsilon_{i}-\mu_{\sigma}$ consisting of the on-site interaction $U$, a local random energy $\epsilon_{i}$, and the chemical potential $\mu_{\sigma} \equiv \mu+\sigma h$, where $h$ is the magnetic field. We wish to calculate the averaged free energy

$$
\Omega_{\mathrm{av}}=-\beta^{-1}\langle\ln \operatorname{Tr} \exp (-\beta \hat{H})\rangle_{\mathrm{av}},
$$

where $\langle\cdots\rangle_{\mathrm{av}}$ is the configurational average over the random energies $\epsilon_{i}$. For this we first have to calculate the partition function for one impurity configuration in $d=\infty$ and then have to average over the disorder. The first step is equivalent to the evaluation of the self-energy $\Sigma_{i j}(z)$ with a complex energy $z$ of an inhomogeneous, interacting quantum system. In the limit $d \rightarrow \infty$ the nonlocal terms in (1) have to be scaled as $t_{\sigma} \rightarrow t_{\sigma}^{*} / \sqrt{2 d}$, and $V^{\sigma \sigma^{\prime}} \rightarrow V^{* \sigma \sigma^{\prime}} / 2 d$ for nearest-neighbor interaction, where $t^{*}$ and $V^{* \sigma \sigma^{\prime}}$ are kept constant. ${ }^{8,9}$ Due to this scaling the self-energies reduce to purely local quantities, i.e., $\Sigma_{i j}(z)=\Sigma_{i i}(z) \delta_{i j}$. However, even this restricted problem cannot be solved exactly, since it would require the calculation of macroscopically many quantities $\Sigma_{i i}(z)$. Due to the local character of $\Sigma$, the original problem of interacting disordered electrons may equally be viewed as a system of noninteracting particles moving in an effective local, random potential

$$
W_{i, \sigma}(z) \equiv \epsilon_{i}+\Sigma_{i i, \sigma}(z),
$$

which now depends on energy and temperature. This enables one to introduce a homogeneous effective potential $\Sigma_{\sigma}(z)$, which exactly replaces the effect of the random potential. We can therefore formally write down the corresponding self-consistent CPA equation for $\Sigma_{\sigma}(z)$

$$
\begin{aligned}
G_{\sigma}(z) & \equiv \int_{-\infty}^{\infty} d E N_{\sigma}(E)\left[z+\mu_{\sigma}-\Sigma_{\sigma}(z)-E\right]^{-1} \\
& =\left\langle\left[G_{\sigma}^{-1}(z)+\Sigma_{\sigma}(z)-W_{i, \sigma}(z)\right]^{-1}\right\rangle_{\mathrm{av}} .
\end{aligned}
$$

Here, $G_{\sigma}(z)$ is the local part of the averaged Green function and $N_{\sigma}(E)$ is the density of states of noninteracting 
$\sigma$ electrons. However, since $W_{i, \sigma}(z)$ is not explicitly known, (2) cannot be solved. Indeed, the solution of the effective-disorder problem requires the knowledge of the local element of the configuration-dependent Green function $G_{i i, \sigma}(z)$. Making use of the Lippmann-Schwinger equation for $G_{i i, \sigma}$ and introducing the coherent potential $\boldsymbol{\Sigma}_{\sigma}(z)$ we have to determine only the effect of the deviations of the random potential $W_{i, \sigma}(z)$ from the coherent potential $\Sigma_{\sigma}(z)$ on the local Green function. ${ }^{14}$ Using the ergodicity property $L^{-1} \sum_{i} G_{i i, \sigma}(z)=G_{\sigma}(z)$, where $L$ is the number of lattice sites, and the fact that in $d \rightarrow \infty$ the off-diagonal terms vanish at least as $1 / \sqrt{d}$, one obtains

$$
\begin{aligned}
\mathcal{S}_{i i, \sigma}^{-1}(z) & \equiv G_{i i, \sigma}^{-1}(z)+\Sigma_{i i, \sigma}(z) \\
& =G_{\sigma}^{-1}(z)+\Sigma_{\sigma}(z)-\epsilon_{i},
\end{aligned}
$$

whose average leads back to (2). Next we calculate the free energy for one configuration, $\Omega^{\text {conf }}$. This can be done in complete analogy to the homogeneous $\operatorname{case}^{10}$ and yields

$$
\begin{aligned}
\beta \Omega^{\mathrm{conf}}= & -\operatorname{tr} \ln \left[\left(\hat{G}^{0}\right)^{-1}-\hat{W}-\hat{V}\right]+\sum_{i \sigma n} \ln G_{i i, \sigma n}^{-1} \\
& -\sum_{i} \ln Z\left\{g_{i i}^{-1}\right\} .
\end{aligned}
$$

Here, $\hat{G}^{0}, \hat{W}$, and $\hat{V}$ are matrices in lattice indices, where
$\left(\hat{G}^{0}\right)_{i j}=G_{i j, \sigma n}^{0}$ is the free propagator, $(\hat{W})_{i j}=W_{i, \sigma n} \delta_{i j}$, and

$$
(\hat{V})_{i j}=L^{-1} \delta_{i j} \sum_{l, \sigma^{\prime}} V^{* \sigma \sigma^{\prime}}\left\langle\hat{n}_{l \sigma^{\prime}}\right\rangle
$$

The index $n$ denotes the Matsubara frequency $\omega_{n}=(2 n+1) \pi \beta^{-1}$ and $\langle\cdots\rangle$ the thermal-expectation value; $\operatorname{tr}$ is the trace as well as sum over $\sigma$ and $n$. The last term on the right-hand side (rhs) of (4) is the free energy of an effective single-site problem ${ }^{10-12}$ with partition function

$$
Z\left\{S_{i i}^{-1}\right\}=\int \mathscr{D} \Psi \mathcal{D} \Psi^{*} \exp A_{\Psi}\left\{\mathcal{S}_{i i}^{-1}\right\}
$$

and the effective action

$$
\begin{aligned}
A_{\Psi}\left\{S_{i i}^{-1}\right\}= & \sum_{\sigma n} \Psi_{\sigma n}^{*} S_{i i, \sigma n}^{-1} \Psi_{\sigma n} \\
& -\frac{U}{2} \sum_{\sigma \neq \sigma^{\prime}} \int_{0}^{\beta} d \tau \Psi_{\sigma}^{*}(\tau) \Psi_{\sigma}(\tau) \Psi_{\sigma^{\prime}}^{*}(\tau) \Psi_{\sigma^{\prime}}(\tau),
\end{aligned}
$$

where $\Psi^{*}$ and $\Psi$ are Grassmann variables. Using (3) we can now average (4) to obtain $\Omega_{\mathrm{av}}$. Due to (3) $A_{\Psi}$ depends on averaged quantities and $\epsilon_{i}$ only. The average of the first term in (4) is exactly given by the CPA expres$\operatorname{sion}^{15}$

$$
-\left\langle\operatorname{tr} \ln \left[\left(\hat{G}^{0}\right)^{-1}-\hat{W}-\hat{V}\right]\right\rangle_{\mathrm{av}}=-\operatorname{tr} \ln \left[\left(\hat{G}^{0}\right)^{-1}-(\Sigma+V) \hat{1}\right]-\sum_{i \sigma n}\left\langle\ln \left[1+G_{\sigma n}\left(\Sigma_{\sigma n}-W_{i, \sigma n}\right)\right]\right\rangle_{\mathrm{av}}
$$

Here, $V \equiv V_{\sigma}=\Sigma_{\sigma^{\prime}} V^{* \sigma \sigma^{\prime}} n_{\sigma^{\prime}}$ is the Hartree potential and $n_{\sigma}=L^{-1} \sum_{i}\left\langle\hat{n}_{i \sigma}\right\rangle$ is the density of $\sigma$ electrons. (Note that due to ergodicity, $n_{\sigma}$ is configuration independent.) Furthermore, using (6) and inserting (3) into the second term on the rhs of (4), the dependence of $\Omega_{\mathrm{av}}$ on $W_{i, \sigma n}$ is found to vanish:

$$
\begin{array}{r}
\Omega_{\mathrm{av}} / L=-\beta^{-1} \sum_{\sigma n} \int d E N_{\sigma}(E) \ln \left[i \omega_{n}+\mu_{\sigma}-\Sigma_{\sigma n}-V_{\sigma}\right. \\
-E]-\frac{1}{2} \sum_{\sigma} V_{\sigma} n_{\sigma} \\
+\beta^{-1} \sum_{\sigma n} \ln G_{\sigma n}^{-1}-\beta^{-1}\left\langle\ln Z\left\{\mathcal{S}_{\sigma}^{-1}-\epsilon_{i}\right\}\right\rangle_{\mathrm{av}},
\end{array}
$$

where $\mathcal{S}_{\sigma}^{-1} \equiv G_{\sigma}^{-1}+\Sigma_{\sigma}$. Expression (7) is the exact free energy of the extended Hubbard model with local disor$\operatorname{der}(1)$ in $d=\infty$. The function $\Sigma_{\sigma n}$ and the parameters $n_{\sigma}$ are determined by $\delta \Omega_{\mathrm{av}} / \delta \Sigma_{\sigma n}=0$ and $\partial \Omega_{\mathrm{av}} / \partial n_{\sigma}=0$, respectively.

Each term of $\Omega_{\mathrm{av}}$ in (7) has a clear mean-field interpretation, which allows us to outline a simple construction of $\Omega_{\mathrm{av}}$ on a mean-field level. We first introduce a homogeneous, ${ }^{16}$ effective energy-dependent potential $\Sigma_{\sigma}(z)$, which is defined in such a way that in the thermodynamic limit the system of disordered, interacting electrons is equivalently described by a system of noninteracting electrons in the potential $\Sigma$; this is schematically shown in Fig. 1(a). The free energy $\Omega_{\mathrm{av}}\{\Sigma\}$ is now constructed as follows [Fig. 1(b)]: (i) we start with the free energy of noninteracting electrons in the potential $\Sigma$ [first two terms in (7)]; (ii) then we remove the potential $\Sigma$ from site $i$, i.e., subtract its energy contribution [third term in (7)]; and (iii) we replace it with the potential operator $\hat{v}_{i \sigma}$, and finally average over $\epsilon_{i}$ [last term in (7)]. ${ }^{17}$ To determine the potential $\Sigma$ we demand that the free energy obtained by this construction be stationary under varying $\Sigma$, i.e., $\partial \Omega_{\mathrm{av}} / \partial \Sigma=0$, which is a self-consistent equation for $\Sigma$ [see Fig. 1(c)].

The self-consistency problem of determining $\Sigma$ is complicated by the competition between the local Hubbard interaction and the kinetic energy of both spins, which causes a nontrivial coupling of the quantum degrees of freedom (Matsubara frequencies) in any dimension. To obtain more explicit insight into the effects caused by the mutual influence of interaction and disorder, we apply the general results to an analytically solvable case, namely, the simplified Hubbard model (SHM), where $V^{* \sigma \sigma^{\prime}}=0$ and $\downarrow$ spins are static, i.e., $t_{\downarrow}=0$, in (1). ${ }^{18}$ In the absence of disorder, this model can be solved exactly in $d=\infty .{ }^{19,20,10}$ The simplification of the kinetic energy 

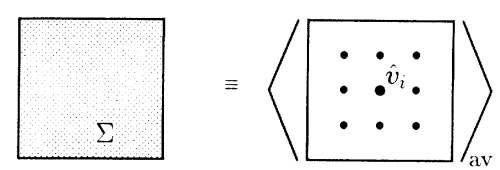

(a)

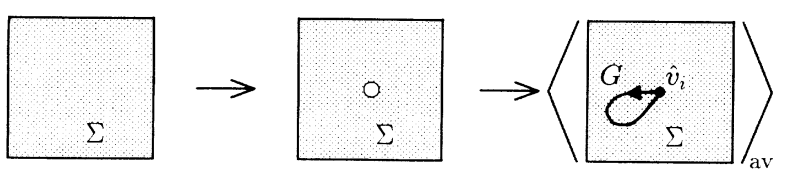

(b)

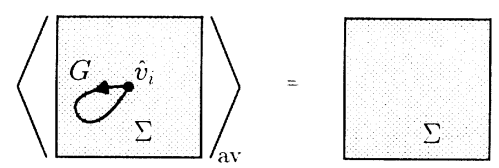

(c)

FIG. 1. (a) Definition of the homogeneous coherent potential $\Sigma$; (b) mean-field construction of the averaged free energy $\Omega_{\mathrm{av}}$ (see text); (c) determination of the potential $\Sigma$ from the stationarity of the process in (b).

implies that $\downarrow$ electrons on site $i$ decouple from the medium $\left(S_{\downarrow n}^{-1}=i \omega_{n}+\mu_{\downarrow}\right)$ and hence can be integrated out. Then (7) reduces to

$$
\begin{aligned}
\Omega_{\mathrm{av}} / L= & -\beta^{-1} \sum_{n}\left\{\int d E N_{\uparrow}(E) \ln \left(i \omega_{n}+\mu_{\uparrow}-\Sigma_{\uparrow n}-E\right)\right. \\
& \left.+\left\langle\ln \left[1+G_{\uparrow n}\left(\Sigma_{\uparrow n}-\epsilon_{i}\right)\right]\right\rangle_{\mathrm{av}}\right\} \\
& -\beta^{-1}\left\langle\ln \left[1+\exp \left\{\beta\left(\mu_{\downarrow}-\epsilon_{i}-\mathscr{E}_{i \uparrow}\right)\right\}\right]\right\rangle_{\mathrm{av}}
\end{aligned}
$$

where

$$
\mathscr{E}_{i \uparrow}=-\beta^{-1} \sum_{n} \ln \left[\Lambda_{n}\left(\epsilon_{i}\right) / \Lambda_{n}\left(\epsilon_{i}+U\right)\right],
$$

with

$$
\Lambda_{n}(x) \equiv\left[1+G_{\uparrow n}\left(\Sigma_{\uparrow n}-x\right)\right]^{-1},
$$

is the energy shift of the $\downarrow$-spin energy due to interaction with $\uparrow$ spins. Stationarity of (8) with respect to $\Sigma_{\uparrow n}$ yields a CPA-type equation for $\Sigma_{\uparrow n}$, namely, (2b) with

$$
W_{i, \sigma n}=\epsilon_{i}+U n_{i \downarrow} \Lambda_{n}\left[\epsilon_{i}+U\left(1-n_{i \downarrow}\right)\right] / \Lambda_{n}\left(\epsilon_{i}\right),
$$

which depends on $U, \beta$ and $\mu_{\sigma}$; here,

$$
n_{i \downarrow}=f\left(\epsilon_{i}+\mathscr{E}_{i \uparrow}-\mu_{\downarrow}\right),
$$

with $f$ as the Fermi function. We thus see that on the level of one-particle Green functions the quantum degrees of freedom are still decoupled as in the limiting cases $\epsilon_{i} \rightarrow 0$, or $U \rightarrow 0$ : the equations for $\Sigma_{\uparrow n}$ have the algebraic CPA structure, i.e., they are diagonal in frequency. In these two limits even higher-order correlation functions are diagonal. However, this no longer holds for two (or more) -particle Green functions when disorder and interactions are present simultaneously. In particu- lar, we will now show that two-particle correlation functions, e.g., for the $\downarrow$-spin density $\chi_{\downarrow}=L^{-1} \sum_{i} \chi_{i \downarrow}$, where $\chi_{i \downarrow}=d n_{i \downarrow} / d \mu_{\downarrow}$ and the staggered density

$$
\chi_{\downarrow}^{\mathrm{stag}}=L^{-1} \sum_{i}(-1)^{i} \chi_{i \downarrow}
$$

are no longer given by algebraic, but by integral, equations. We first calculate $\chi_{\downarrow}$. From the definition of $n_{i \downarrow}$ and the self-energy $\Sigma_{\uparrow n}$ in (2), we get

$$
\begin{aligned}
\chi_{\downarrow} \equiv\left\langle\chi_{i \downarrow}\right\rangle_{\mathrm{av}}=\left\langle\chi_{i \downarrow}^{0}\right\rangle_{\mathrm{av}}-U^{2} \beta^{-1} \sum_{n} & \left\langle\chi_{i \downarrow}^{0} X_{i n}\right\rangle_{\mathrm{a} v} \mathcal{K}_{n} \\
& \times\left\langle X_{i n} \chi_{i \downarrow}\right\rangle_{\mathrm{av}},
\end{aligned}
$$

where $\chi_{i \downarrow}^{0}=\beta n_{i \downarrow}\left(1-n_{i \downarrow}\right)$. Here,

$$
X_{i n}=G_{\uparrow n} \Lambda_{n}\left(\epsilon_{i}\right) \Lambda_{n}\left(\epsilon_{i}+U\right)
$$

is a renormalized local propagator, and $\mathcal{K}_{n}=g_{n}$ / $\left(1-g_{n} \Gamma_{n}\right)$, with $g_{n}=1-G_{\uparrow n}^{2} /\left\langle G_{\uparrow n}^{2}\right\rangle$,

$$
\Gamma_{n}=\left\langle\left(1-n_{i \downarrow}\right) \Lambda_{n}^{2}\left(\epsilon_{i}\right)+n_{i \downarrow} \Lambda_{n}^{2}\left(\epsilon_{i}+U\right)\right\rangle_{\mathrm{av}},
$$

and

$$
\left\langle G_{\uparrow n}^{2}\right\rangle \equiv \int d E N_{\uparrow}(E)\left[i \omega_{n}+\mu_{\uparrow}-\Sigma_{\uparrow n}-E\right]^{-2}
$$

is the dimensionless two-particle correlation function. Note that $\chi_{\downarrow}^{\text {stag }}$ is also given by (9), where now

$$
\left\langle G_{\uparrow n}^{2}\right\rangle \equiv G_{\uparrow n} /\left(i \omega_{n}+\mu_{\uparrow}-\Sigma_{\uparrow n}\right) .
$$

Obviously, (9) is not a closed equation for the averaged function $\left\langle\chi_{i \downarrow}\right\rangle_{\mathrm{av}}$, since on the rhs a new quantity $\left\langle X_{i n} \chi_{i \downarrow}\right\rangle_{\text {av }}$ appears which cannot be directly obtained from $\Omega_{\mathrm{av}}$. Hence, we must go back to the configurationdependent free energy (4) to determine $X_{\text {in }} \chi_{i \downarrow}$. Having averaged the corresponding equation, we obtain a linear integral equation

$$
\begin{aligned}
\left\langle X_{i n} \chi_{i \downarrow}\right\rangle_{\mathrm{av}}= & \left\langle X_{i n} \chi_{i \downarrow}^{0}\right\rangle_{\mathrm{av}} \\
& -U^{2} \beta^{-1} \sum_{n^{\prime}}\left\langle X_{i n} \chi_{i \downarrow}^{0} X_{i n^{\prime}}\right\rangle_{\mathrm{av}} \mathcal{K}_{n^{\prime}}\left\langle X_{i n^{\prime}} \chi_{i \downarrow}\right\rangle_{\mathrm{av}},
\end{aligned}
$$

which now fully determines the quantity $\left\langle X_{i n} \chi_{i \downarrow}\right\rangle_{\mathrm{av}}$. Hence, contrary to the case of the one-particle Green function, the quantum degrees of freedom are seen to be coupled on the level of two-particle correlation functions. This can be expected to lead to new phases and phase transitions that have no counterparts in noninteracting, disordered, or pure, interacting systems. Note that this effect requires the self-energy for the nonrandom model to be frequency dependent and hence is lost in any Hartree-Fock treatment.

The explicit form of the integral equation (10) depends on the distribution of random energies. For a binary alloy, where only two energy levels exist [e.g., $\epsilon_{i}=0, \Delta$ with probabilities $(1-x)$ and $x$, respectively], (10) reduces to a $2 \times 2$ matrix equation. Except for half filling the equation for the critical temperature, $\chi_{\downarrow}^{-1}=0$, allows for two solutions, i.e., one has a reentrant behavior at low temperatures. At half filling on a bipartite lattice one always has 
an ordered phase with checkerboard structure with $T_{c} \propto(1-x) T_{c}^{0}$, where $T_{c}^{0}$ is the critical temperature of the pure model.

Finally, we address the structure of the ordered phase in the presence of disorder. The free energy of the SHM in the checkerboard $(A-B)$ phase $\Omega_{\mathrm{av}}^{A B}$ can be obtained from (8) as in the pure case, ${ }^{10}$ by introducing $\Sigma_{\uparrow}^{\alpha}, \alpha=A, B$. There is, however, an important difference between pure and random cases. Denoting the averaged density of $\downarrow$ electrons on a sublattice by $n_{\downarrow}^{\alpha}=\left\langle n_{i \downarrow}^{\alpha}\right\rangle_{\mathrm{av}}$, a single-order parameter $\Delta n_{\downarrow}=n_{\downarrow}^{A}-n_{\downarrow}^{B}$ is sufficient to describe the ordered phase in the pure case. This is no longer true in the presence of disorder. In particular, the last term on the rhs of (8) then takes the form

$$
\begin{aligned}
\frac{1}{2 \beta} \sum_{\alpha=A, B}\left\langle\left(1-n_{i \downarrow}^{\alpha}\right) \ln \left(1-n_{i \downarrow}^{\alpha}\right)+n_{i \downarrow}^{\alpha} \ln n_{i \downarrow}^{\alpha}\right. \\
\left.+\left(\epsilon_{i}-\mu_{\downarrow}+\mathscr{E}_{i \uparrow}^{\alpha}\right) n_{i \downarrow}^{\alpha}\right\rangle_{\mathrm{av}} .
\end{aligned}
$$

It is clear that, due to the presence of arbitrary powers of $n_{i \downarrow}^{\alpha}, \Omega_{\mathrm{av}}^{A B}$ is not only a function of the single parameter $\Delta n_{\downarrow}$ but depends on an infinite number of averaged quantities $\left\langle\left(n_{i \downarrow}^{\alpha}\right)^{k}\right\rangle_{\text {av }}$ with $k=1,2, \ldots$. This is reminiscent of the configuration-dependent mean-field free energy of Thouless, Anderson, and Palmer in the classical spinglass problem. ${ }^{21}$ Due to this fact, we expect that the low-temperature phases of disordered, interacting lattice electron systems have a much more complex structure than pure systems.

In summary, we derived the exact averaged free energy for an extended Hubbard model with local disorder in $d=\infty$. We thereby obtained a thermodynamically consistent, nonperturbative description of this general class of models on a mean-field level. The free-energy expression (7) offers a clear interpretation in terms of a single site embedded in a homogeneous effective medium. Whenever an exact or approximate solution of the nonrandom model in $d=\infty$ is given, the additional effect of disorder is obtained exactly from (7). In the case of the simplified Hubbard model, we obtained analytic expressions for the averaged free energy, as well as for one- and two-particle correlation functions. The simultaneous presence of interactions and disorder leads to a new coupling of the quantum degrees of freedom in two-particle quantities that has no counterpart in noninteracting, disordered, or interacting, pure systems. The exact solution in $d=\infty$, in conjunction with a $1 / d$ expansion, provides a suitable starting point for a systematic investigation not only of the thermodynamic properties, but-in view of the striking analogy to the solution for the classical spin-glass model in $d=\infty$ - even of such effects as ergodicity breaking and Anderson localization.

This work was supported in part by the Sonderforschungsbereich 341 of the Deutsche Forschungsgemeinschaft.
${ }^{1}$ P. A. Lee and T. V. Ramakrishnan, Rev. Mod. Phys. 57, 287 (1985).

${ }^{2}$ A. M. Finkelstein, Zh. Eksp. Teor. Fiz. 84, 166 (1983) [Sov. Phys. JETP 57, 97 (1983)]; Z. Phys. B 56, 189 (1984).

${ }^{3}$ C. Castellani, C. DiCastro, P. A. Lee, and M. Ma, Phys. Rev. B 30, 527 (1984); C. Castellani, G. Kotliar, and P. A. Lee, Phys. Rev. Lett. 56, 1179 (1987).

${ }^{4}$ D. Belitz and T. Kirkpatrick, Phys. Rev. Lett. 63, 1296 (1989); Physica A 167, 259 (1990).

${ }^{5}$ M. Milovanović, S. Sachdev, and R. N. Bhatt, Phys. Rev. Lett. 63, 82 (1989).

${ }^{6}$ M. Ma, Phys. Rev. B 26, 5097 (1982).

${ }^{7}$ G. Zimanyi and E. Abrahams, Phys. Rev. Lett. 64, 2719 (1990).

${ }^{8}$ W. Metzner and D. Vollhardt, Phys. Rev. Lett. 62, 324 (1989).

${ }^{9}$ E. Müller-Hartmann, Z. Phys. B 74, 507 (1989).

${ }^{10}$ V. Janiš, Z. Phys. B 83, 227 (1991).

${ }^{11}$ A. Georges and G. Kotliar, Phys. Rev. B 45, 6479 (1992).

${ }^{12}$ V. Janiš and D. Vollhardt, Int. J. Mod. Phys. B 6, 731 (1992).
${ }^{13}$ R. Vlaming and D. Vollhardt, Phys. Rev. B 45, 4637 (1992).

${ }^{14} \mathrm{G}$. Rickayzen, Green's Functions and Condensed Matter (Academic, London, 1984).

${ }^{15}$ V. Janiš, Phys. Rev. B 40, 11331 (1989).

${ }^{16}$ In the case of a broken symmetry we must introduce more than one local effective potential.

${ }^{17}$ Note that the energy at site $i$ is measured relative to the surrounding medium; that is why the effective local propagator of electrons at site $i$ is given as $S=\left[G^{-1}+\Sigma\right]^{-1}$.

${ }^{18}$ Another solvable case is that of spinless fermions, which is discussed by R. Vlaming, G. Uhrig and D. Vollhardt, J. Phys. Condens. Matter 4, 7773 (1992).

${ }^{19}$ U. Brandt and C. Mielsch, Z. Phys. B 75, 365 (1989); 79, 295 (1990); 82, 37 (1991).

${ }^{20}$ P. van Dongen and D. Vollhardt, Phys. Rev. Lett. 65, 1663 (1990).

${ }^{21}$ D. J. Thouless, P. W. Anderson, and R. G. Palmer, Philos. Mag. 35, 593 (1977). 

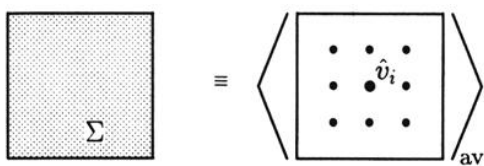

(a)

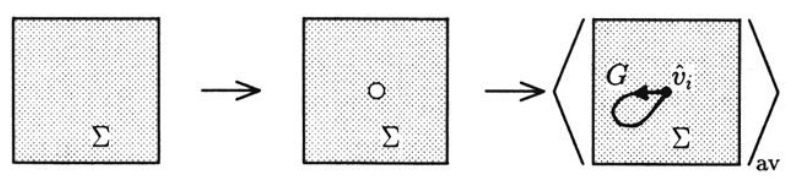

(b)

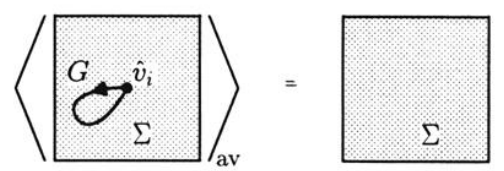

(c)

FIG. 1. (a) Definition of the homogeneous coherent potential $\Sigma$; (b) mean-field construction of the averaged free energy $\Omega_{\mathrm{av}}$ (see text); (c) determination of the potential $\Sigma$ from the stationarity of the process in (b). 